\title{
ESTRATEGIAS CLAVE PARA IMPLEMENTAR UN SISTEMA DE GESTIÓN DE LA INNOVACIÓN EN PYMES DEL SECTOR TEXTIL, CONFECCIÓN, DISEÑO Y MODA DEL MUNICIPIO DE ITAGÜÍ- ANTIOQUIA- COLOMBIA
}

\section{KEY STRATEGIES TO IMPLEMENT A SYSTEM OF INNOVATION MANAGEMENT IN SMES OF THE TEXTILE SECTOR, CLOTHING, DESIGN AND FASHION OF THE MUNICIPALITY OF ITAGÜÍ- ANTIOQUIA- COLOMBIA}

\author{
Alicia Magnolia Vásquez Franco ${ }^{1}$ \\ Wilson Alberto Cely Acevedo² \\ Martha de Jesús Guerrero Guzmán³
}

\section{Resumen}

Las Pymes representan un excelente medio para impulsar el desarrollo económico del país, razón por la cual es importante investigar acerca de los procesos de innovación, capacidad tecnológica y recursos económicos que permitan hacer de las Pymes del sector textil, específicamente de la confección y la moda, industrias prósperas con alto nivel de competitividad, tanto nacional como internacionalmente.

El propósito de la presente investigación es el análisis de la innovación en las pequeñas y medianas empresas (pymes) del sector textil, confección, diseño y moda del municipio de Itagüí - Antioquia- Colombia y cómo ésta puede ser un factor

\footnotetext{
${ }^{1}$ Profesional en ciencias administrativas, maestrante en Administración de organizaciones 2 Ingeniero agropecuario, maestrante en Administración de Organizaciones

${ }^{3}$ Docente Universidad Nacional Abierta y a Distancia. Magíster en administración de empresas y liderazgo estratégico.
} 


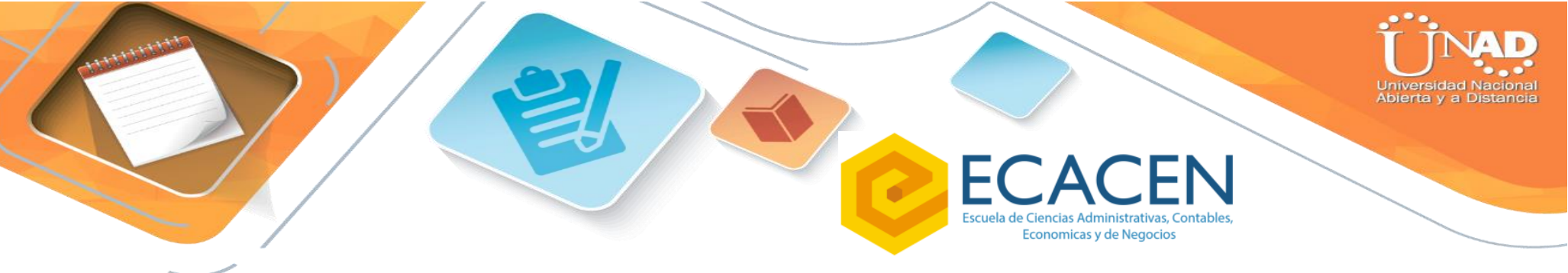

De acuerdo al Departamento Nacional de Planeación DNP, (2007), en Colombia las Pymes en especial las del sector textil, son una de las principales fuentes de generación de empleo, además que dinamizan la economía, razón por la cual cumplen un papel de tipo social, sobre todo en la disminución de pobreza y mejoramiento de calidad de vida en las zonas donde tienen afectación. Sin embargo, y tal como lo menciona Gaviria (2015), es evidente que estas organizaciones requieren adaptarse al cambio y enfrentar los desafíos de la intensa competencia de los mercados globalizados, las teorías administrativas, la gestión de las operaciones, las estrategias de mercado, la innovación, los modelos de negocio, etc.

Es esencial que cada empresa (pyme) cree y fomente esa cultura para aprovechar mejor sus recursos, liderar procesos y procedimientos y estar un paso adelante ante los cambios que se dan en un mundo global cada vez más versátil y competido, buscando la excelencia y posicionando la organización, tratando de mejorar y sobrepasar estándares de talla internacional.

El presente artículo resume la investigación sobre la adopción de estrategias adecuadas para lograr implementar un sistema de gestión de la innovación en las pymes del sector textil, confección, diseño y moda del municipio de Itagüí- AntioquiaColombia.

\section{Importancia de la Innovación}

Para que una organización sea exitosa y pueda permanecer en el tiempo, debe emplear correctamente todas las herramientas de que dispone como las tecnologías de la información y las comunicaciones, el talento humano y el mercado. De igual manera y no menos importante es el conocimiento y la forma como éste se gestiona al interior de la organización. 


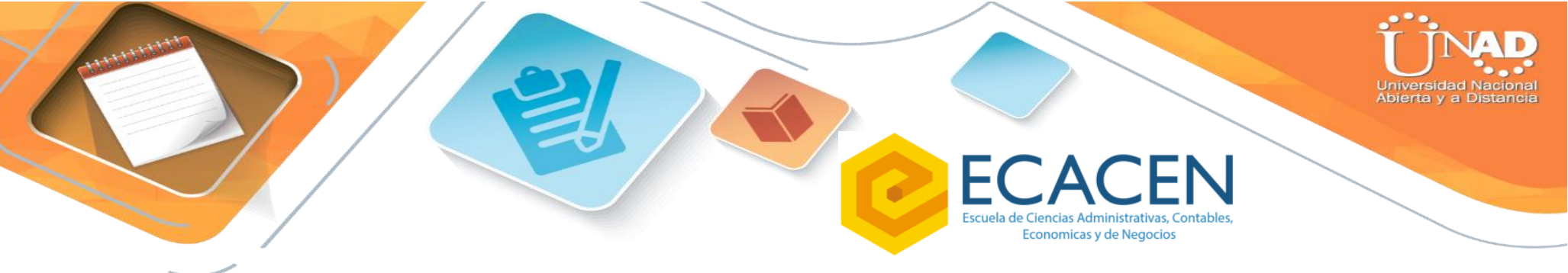

De acuerdo a Ponjuan-Dante (2006), "la gestión del conocimiento es como una cultura al interior de la organización que busca ante todo aplicar ese conocimiento para que esta sea más productiva y sobre todo competitiva".

Torres y Santa (2013), afirman que la innovación es una determinante de la actividad empresarial y que ésta no es solo tecnológica, sino que requiere de una visión multidisciplinar. Los autores complementan afirmando que se necesita de una constante observación y análisis del entorno (científico y tecnológico), en las universidades, las empresas, el medio social.

De acuerdo a Quintero (2016), para lograr el desarrollo sostenible de las empresas se requiere de una integración económica del conocimiento (innovación) + tecnología. De igual forma señala, que en Colombia solo el 5\% de las Mipymes son exportadoras, mientras que en Latinoamérica el promedio es del $10 \%$ y en Europa este llega al $40 \%$.

Según los autores, es aplicando creativamente, tal como lo menciona el profesor Landes, y de manera estratégica las oportunidades que brinda la tecnología en el mundo de los negocios, muchas empresas usan las Tics en forma innovadora para originar y sustentar ventajas competitivas y así aprovechar las condiciones de cambio continuo en el mercado.

\section{Antecedentes de Investigación}

Una herramienta que a nuestro juicio es fundamental para el fortalecimiento y posicionamiento de cualquier empresa es la innovación. De por sí, estamos ante un concepto que ha evolucionado en el tiempo de acuerdo a múltiples factores, pero que siempre ha tratado de conservar su esencia, lo novedoso. 


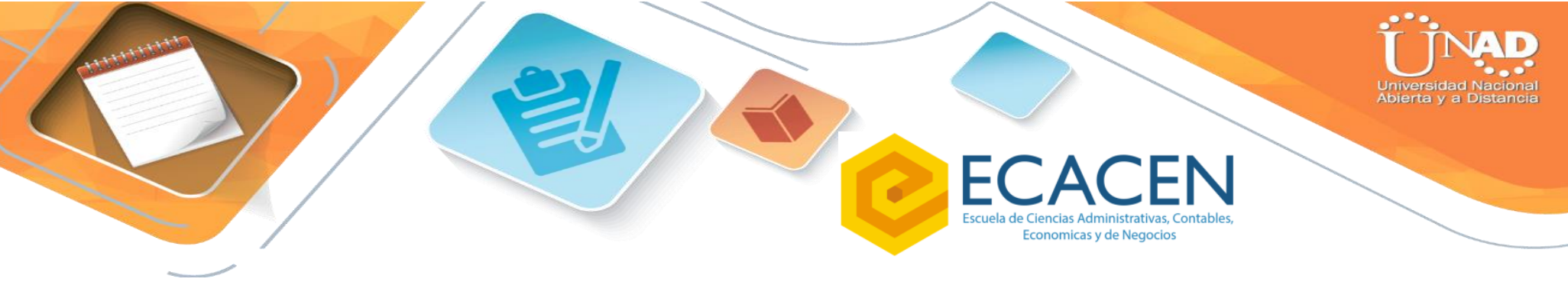

Para el año 2015, Colombia se posiciona en el puesto 67 y a nivel de Latinoamérica se ubica en la posición No 6, por debajo de Barbados, Chile, Costa Rica, México y Panamá.

Según lo planteado en el Manual de Oslo, Innovación es la implementación de un producto (bien o servicio) o proceso nuevo o con un alto grado de mejora, o un método de comercialización u organización nuevo aplicado a las prácticas de negocio, al lugar de trabajo o a las relaciones externas. OCDE, 2005, p.33.

\section{Importancia de la innovación}

La innovación permite a las personas y a las empresas propiciar escenarios de emprendimiento, transformando el conocimiento en nuevos productos, lo que permite modificar las organizaciones y plantear nuevos y prósperos escenarios económicos, es de anotar que el verdadero empresario es una persona con características acentuadas en liderazgo y emprendimiento, capaz de fomentar la creatividad y la innovación contribuyendo a generar e implementar nuevos productos y nuevos procesos, en muchos casos ambicioso y seguro con el ánimo de generar riqueza y estabilidad económica.

Según Drucker (1985), en su libro "Innovation and Etrepreneurship", dice "Las innovaciones no tienen valor según su novedad, contenido científico o ingenio de la idea sino por su éxito en el mercado. La innovación ocurre cuando los emprendedores crean nuevos productos, abren otras áreas de negocio, cuando crean nuevas empresas completamente nuevas generan bienestar para muchas personas creando nuevos puestos de trabajo y pagando más impuestos". 


\section{Elementos a tener en cuenta en un proceso de innovación}

Tabla 1. Elementos para un Plan de Innovación

Elementos

\section{Detalle}

Declaración de la importancia de la Definir por qué la empresa desea iniciar un Innovación para la empresa. programa para sistematizar la innovación.

Establecer la definición operativa que se utilizará para evaluar si algo es innovador o no.

Definir una visión de la innovación para describir a donde se desea llegar a través de la innovación.
Definición de los objetivos y metas de la Innovación.
Definir los resultados específicos medibles que se espera alcanzar con la innovación.
Establecimiento de una estrategia y vectores de Innovación.
Determinar de qué manera se espera enfocar la innovación y alinearla con la estrategia empresarial.

Determinar cuáles son los esfuerzos de innovación que se realizarán durante el año. ideas y proyectos de Innovación.

Establecer las pautas necesarias para determinar el retorno de la inversión del proyecto de innovación.

Fuente: Morales y León 2013 
Tabla 2. La Innovación como proceso de gestión integral

\begin{tabular}{|c|c|}
\hline $\begin{array}{l}\text { Propósito } \\
\text { motivador y } \\
\text { valores }\end{array}$ & $\begin{array}{l}\text { Las compañías centradas en la innovación son lugares de } \\
\text { trabajo inspiradores. Valores como integridad, confianza, } \\
\text { liderazgo y pasión se traducen en la acción y se demuestran en } \\
\text { los comportamientos cotidianos. }\end{array}$ \\
\hline Metas estrechas & $\begin{array}{l}\text { Identificar unas pocas metas críticas crea claridad para } \\
\text { focalizar estrategias ganadoras que alinean la energía de todos }\end{array}$ \\
\hline $\begin{array}{l}\text { Estrategias a } \\
\text { elección }\end{array}$ & $\begin{array}{l}\text { Una vez que se establecen las metas, es necesario delinear } \\
\text { como lograrlas. Las elecciones que resulten permitirán ganar } \\
\text { con los consumidores y contra la competencia. }\end{array}$ \\
\hline $\begin{array}{l}\text { Fortalezas } \\
\text { esenciales únicas }\end{array}$ & $\begin{array}{l}\text { Una vez que se realizaron las elecciones respecto de donde } \\
\text { jugar, es preciso concentrarse en como gana, construyendo a } \\
\text { partir de las fortalezas. }\end{array}$ \\
\hline $\begin{array}{l}\text { Estructuras } \\
\text { posibilitadoras }\end{array}$ & Es necesario diseñar estructuras y procesos confortables. \\
\hline $\begin{array}{l}\text { Sistemas } \\
\text { consistentes y } \\
\text { confiables }\end{array}$ & $\begin{array}{l}\text { La innovación es creativa pero no caótica. Es una vía } \\
\text { sistemática para moverse desde el concepto hacia la } \\
\text { comercialización. }\end{array}$ \\
\hline $\begin{array}{l}\text { Cultura audaz y } \\
\text { conectada }\end{array}$ & $\begin{array}{l}\text { Una cultura es lo que la gente hace todos los días sin que se lo } \\
\text { digan. En una compañía centrada en la innovación, los } \\
\text { managers y empleados no temen a la innovación. }\end{array}$ \\
\hline $\begin{array}{l}\text { Liderazgo } \\
\text { inspirador }\end{array}$ & $\begin{array}{l}\text { Ninguna organización puede trabajar si líderes. En un proceso } \\
\text { integrado de innovación, los líderes articulan todos los }\end{array}$ \\
\hline
\end{tabular}




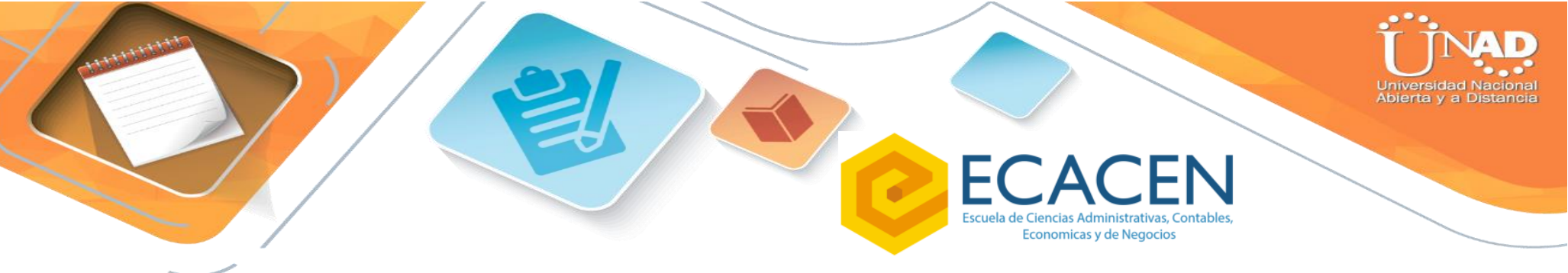

- Tecnología y conocimientos propios.

- Redes establecidas y sistema de innovación

- Innovación Incremental (I): Más de lo mismo, pero mejor.

- Innovación Sustancial (S): Cosas diferentes, aunque es difícil sostenerse en el tiempo.

- Innovación Radical (R): Resultados distintos para la industria.

\section{Estrategias claves para la Innovación}

- Equipo humano con actitud innovadora

- Observación y análisis del entorno

- Cultura organizacional innovadora

- Interdisciplinariedad del equipo

- Vigilancia tecnológica e Inteligencia competitiva

- Análisis prospectivo

- Acción

\section{La Creatividad}

Mario Morales en su libro "Adiós a los mitos de la Innovación", la mayoría de las personas confunden creatividad con innovación, asumiendo que son lo mismo, pero la diferencia radica en que:

\section{Creatividad es pensar nuevas ideas}

Innovación es implementar una nueva idea para crear valor

Según G. Wallas (1926), el Número de etapas que contribuyen en el proceso creativo que postuló en su libro "El arte del pensamiento" (1926) son 4 que se re realimentan entre sí creando un bucle. 


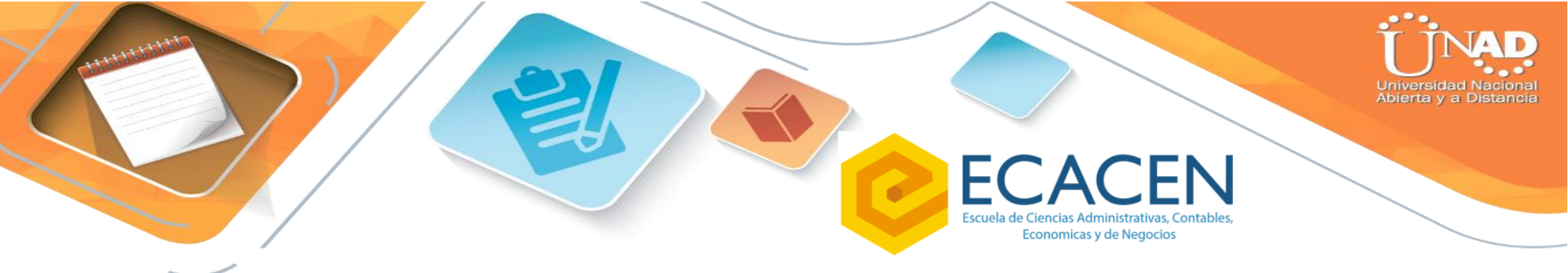

manejo de la información interna de las empresas, la VT es la que se encarga de analizar la información externa.

\section{Cultura organizacional innovadora}

Una empresa que desea implementar la innovación debe asegurar los procedimientos, herramientas e instrumentos necesarios para generar conocimiento y detectar oportunidades: creación grupos de trabajo, sesiones que fomenten el trabajo en equipo, la creatividad, propuesta, etc.

- Biodireccionalidad de la comunicación: La fórmula más fiable para asegurar el éxito de la innovación es estar alerta a las señales del entorno.

- Incentivar el talento: Un sistema de incentivos adecuado favorece el entorno innovador al interior de la organización.

- Acciones de seguimiento y evaluación del impacto:Todo proyecto de innovación debe acompañarse de ciertas actividades de seguimiento, sesiones de formación, análisis del impacto y evaluación del valor aportado.

- Estructuras organizativas horizontales: Permite a los recursos humanos actuar con más libertad y poder de decisión. Sin embargo, la dirección siempre debe mantener mecanismos de control y herramientas que permitan evaluar qué proyectos de innovación son los que poseen más valor agregado, con el fin de asignar el recurso y el presupuesto necesario para su ejecución.

\section{Conclusiones}

Uno de los elementos importantes a tener en cuenta a la hora de hablar de innovación en pymes, es la poca importancia que estas le dan al conocimiento y su relevancia dentro del proceso de innovación. La gestión de conocimiento al interior de 


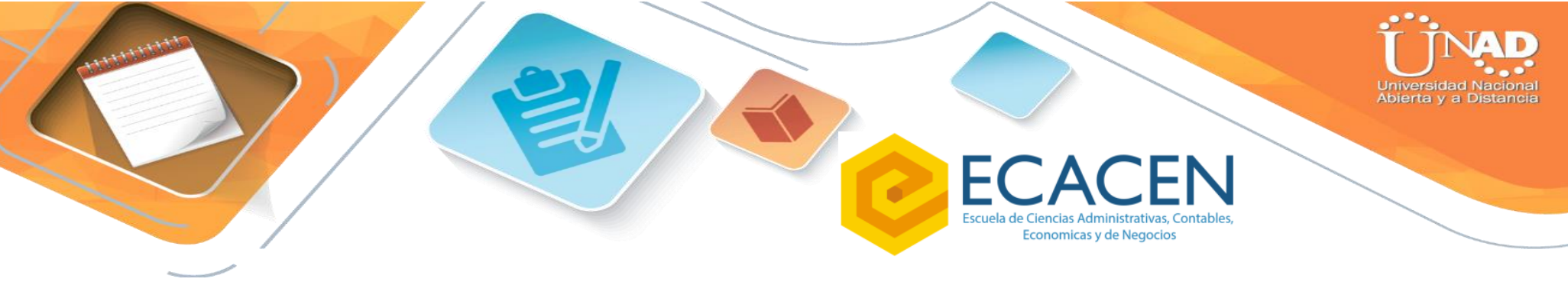

la organización, le permite tener la capacidad de adaptarse mejor a los cambios tan turbulentos de la actualidad.

El activo más importante para cualquier organización es su capital humano, a su vez, el empresario es quien lo representa y por tal motivo, es quien debe promover la generación de conocimiento. Este conocimiento debe ser el motor que le permita adaptarse y permanecer en el mercado, ya sea en un contexto de globalidad o de proteccionismo.

Como se mencionó anteriormente, el conocimiento es la clave, y su generación son la base para todo lo que cualquier organización deba hacer en materia de innovación; la generación de conocimiento inicia con una idea creativa, por tal motivo, toda pyme debe propiciar ambientes para la creación, aplicación y difusión de esas ideas que marcan diferencia y permiten el desarrollo económico de la organización.

Una manera de crear conocimiento, sobre todo cuando no se dispone de medios, recursos, voluntad, o apatía por parte de los empresarios y gerentes de las pymes, es que estos recurran a la academia. Las universidades, los centros de investigación, podrían ser el punto de encuentro, donde las pymes colocan sus inquietudes en la mesa, mientras que la academia, puede contar con el personal idóneo, capacitado y dispuesto a trabajar en equipo, darán las respuestas a inquietudes manifiestas, siempre y cuando se dan las condiciones adecuadas.

Toda empresa u organización cuando desea innovar, es porque se ha dado cuenta que existe una demanda o necesidad insatisfecha, en algunos casos porque constantemente están saliendo nuevos productos o servicios, en otros porque lo que hacen, lo hacen o muy lento, o no cuentan con la tecnología adecuada, puede ser tomado como disculpa, o porque hay un desconocimiento de ciertos indicadores que le permitan ser competitivos. 


\section{Referencias}

Araujo, A. (2010). La cultura organizacional innovadora desde una perspectiva valorativa. Visión gerencial 9 (2) 219 - 229 Universidad de los Andes.

Departamento Nacional de Planeación DNP, (2007),

Drucker, P. F. (1985). The discipline of innovation. Harvard business review, 63(3), 67-72.

Gaviria, J., (2015). El impacto de internet en las Mipymes del sector textil confección. En Antioquia. Tesis. Universidad Eafit. Medellín.

González y García. (2010). Innovación abierta: Un modelo preliminar desde la gestión del conocimiento. Intangible capital. 7(1), 82-115.

G. Wallas, G. (1926) El arte del pensamiento. Etapas que contribuyen en el proceso creativo. libro 6

Lafley, A. G., \& Charan, R. (2008). The game-changer: How you can drive revenue and profit growth with innovation. Crown Business.

Manual, D. O. (2005). Guía para la recogida e interpretación de datos sobre innovación. Organización para la cooperación y el desarrollo económico (OCDE), 23-24.

Morales, M., León, A.2013. Adiós a los mitos de la Innovación: Una guía práctica para implementar la Innovación en América Latina. Innovare.

Morcillo, P. (2003). Vigilancia e Inteligencia Competitiva: Fundamentos e implicaciones. Revista Tribuna de Debate 17, 
Ponjuán-Dante, G. (2006). Introducción a la gestión del conocimiento. La Habana: Editorial Félix Varela, 10.

Quintero, R. (2016). Innovación el reto de las Mipymes en Colombia. Simposio llevado a cabo en Barranquilla. Agosto 2 al 3.

Rojas, J. 2010. La vigilancia tecnológica como herramienta de competitividad e innovación. Éxito Empresarial. (54).

Torres, R; Santa, A 2013. Estructuras, procesos e instrumentos de vigilancia tecnológica. La vigilancia tecnológica como proceso de innovación relacional Universidad-Empresa Universidades, 63 (58) 33-42. 